\title{
THREE MODELS FOR TWO PHASE FLOW IN POROUS MEDIA
}

\author{
N. H. RISEBRO
}

\begin{abstract}
We compare three different models of two phase flow in a porous medium; the standard Darcy/Buckley-Leverett model, the Brinkman model and the Helmholtz model. These three models are all singular perturbations of the inviscid Darcy model, and thus have the same formal limits. The existence of such limits have not been proved mathematically, and in this paper we investigate numerically whether limits exist, and whether they are similar.
\end{abstract}

\section{THE MACROSCOPIC MODELS}

In this article, a (macroscopic) porous medium is defined through its porosity and permeability. If $\boldsymbol{x}$ denotes the spatial coordinate (usually in $\mathbb{R}^{d}$ for $d=1,2,3$, we shall assume that the porous medium is fixed in time, so that the porosity and permeability do not depend on time. The porosity is denoted by $\phi(\boldsymbol{x})$, and represents the available pore volume. Thus if the medium is not porous at $\boldsymbol{x}, \phi(\boldsymbol{x})=$ 0 , while if it is extremely porous $\phi(\boldsymbol{x}) \approx 1$. Roughly speaking the permeability measures the tendency of fluids to move through the porous medium in different directions. The precise definition of the permeability depends on the model. For simplicity and clarity of exposition, whenever possible, we shall assume that the permeability is 1 .

We shall derive some commonly used idealized models of two phase flow in a porous medium. We assume that we have a porous medium containing two immiscible phases, for simplicity we call these oil and water. The primary quantities of interest here are the saturations of of each phase. The saturation of a phase is defined to be the fraction of the available pore volume occupied by that phase. So if $s_{\mathrm{w}}$ denotes the saturation of water, and $s_{\mathrm{o}}$ that of oil, we have

$$
s_{\mathrm{O}}+s_{\mathrm{w}}=1 \text {. }
$$

If $\boldsymbol{v}_{i}, i=\mathrm{o}, \mathrm{w}$, denotes the velocity of phase $i$, the two phases are transported by their velocities, and conservation of each phase is described by

$$
\left(\phi s_{i}\right)_{t}+\operatorname{div}_{\boldsymbol{x}}\left(\boldsymbol{v}_{i}\right)=0 .
$$

In order to focus on the two phase aspect of these models, we assume that $\phi(\boldsymbol{x})=\phi_{0}$, where $\phi_{0}$ is a positive constant, then we use units such that $\phi_{0}=1$. Using (1.1), we can add (1.2) for $i=\mathrm{w}$, o to get

$$
\operatorname{div}_{\boldsymbol{x}}\left(\boldsymbol{v}_{T}\right)=0, \quad \boldsymbol{v}_{T}=\boldsymbol{v}_{\mathrm{o}}+\boldsymbol{v}_{\mathrm{w}}
$$

The quantity $\boldsymbol{v}_{T}$ is called the total velocity.

Date: May 13, 2019

2010 Mathematics Subject Classification. Primary: 76S05, 65M06; Secondary: 35G61.

Key words and phrases. Darcy's law, Brinkman equation, Helmholtz regularization, numerical methods.

The author has received funding from the European Union's Horizon 2020 research and innovation programme under the Marie Skłodowska-Curie grant agreement No 642768. 
1.1. The standard models. The phase velocities in an isotropic homogeneous porous medium are often assumed to obey Darcy's law for two phase flow [22],

$$
\boldsymbol{v}_{i}=-\lambda_{i} \nabla_{\boldsymbol{x}} p_{i}+\lambda_{i} \rho_{i} g \boldsymbol{k},
$$

where $g$ is the constant gravitational acceleration, $\boldsymbol{k}$ is a unit vector pointing "downwards" and $\rho_{i}$ denotes the constant density of phase $i$. The quantity $\lambda_{i}=\lambda_{i}\left(s_{i}\right)$ is called the relative permeability of phase $i$, and $p_{i}$ is the pressure of phase $i$. We shall assume that the relative permeabilities are known functions with some given properties, but $\lambda_{i}$ is often determined experimentally, assuming that (1.4) holds.

Since we can eliminate one of the saturations we use only the water saturation and write $s=s_{\mathrm{w}}$. Then the total velocity reads

$$
\boldsymbol{v}_{T}=-\left(\lambda_{\mathrm{w}}(s) \nabla_{\boldsymbol{x}} p_{\mathrm{w}}-\lambda_{\mathrm{w}}(s) \rho_{\mathrm{w}} G+\lambda_{\mathrm{o}}(s) \nabla_{\boldsymbol{x}} p_{\mathrm{o}}-\lambda_{\mathrm{w}}(s) \rho_{\mathrm{o}} G\right),
$$

with $G=g \boldsymbol{k}$. Introducing the capillary pressure $p_{\mathrm{cow}}=p_{\mathrm{o}}-p_{\mathrm{w}}$, and assuming that this is a known function of the saturation, we then define the so-called complementary pressure, see [1],

$$
p_{\mathrm{c}}(s)=\int_{0}^{s} \frac{\lambda_{\mathrm{w}}(\sigma)}{\lambda_{\mathrm{w}}(\sigma)+\lambda_{\mathrm{o}}(\sigma)} \frac{\partial p_{\mathrm{cow}}}{\partial s}(\sigma) d \sigma .
$$

We shall define

$$
\lambda_{T}(s)=\lambda_{\mathrm{w}}(s)+\lambda_{\mathrm{o}}(s) \text { and } f(s)=\frac{\lambda_{\mathrm{w}}(s)}{\lambda_{T}(s)} .
$$

With this notation we have that

$$
\nabla_{\boldsymbol{x}} p_{\mathrm{c}}=f(s)\left(\nabla_{\boldsymbol{x}} p_{\mathrm{o}}-\nabla_{\boldsymbol{x}} p_{\mathrm{w}}\right) .
$$

Now we introduce the so-called global pressure $p$ by $p_{\mathrm{o}}-p_{\mathrm{c}}$, so that the total velocity is written

$$
\boldsymbol{v}_{T}=-\lambda_{T} \nabla_{\boldsymbol{x}} p+\left(\lambda_{\mathrm{w}} \rho_{\mathrm{w}}+\lambda_{\mathrm{o}} \rho_{\mathrm{o}}\right) G .
$$

The condition (1.3) reads

$$
-\operatorname{div}_{\boldsymbol{x}}\left[\lambda_{T}(s) \nabla_{\boldsymbol{x}} p-\left(\lambda_{\mathrm{w}}(s) \rho_{\mathrm{w}}+\lambda_{\mathrm{o}}(s) \rho_{\mathrm{o}}\right)\right]=0 .
$$

This is a second order equation for the (global) pressure. We assume that $\lambda_{\mathrm{w}}(s)$ is a continuously non-decreasing function with $\lambda_{\mathrm{w}}(0)=0$ and $\lambda_{\mathrm{W}}(1)=1$, and $\lambda_{\mathrm{o}}(s)$ is a non-increasing function of $s$ such that $\lambda_{\mathrm{o}}(0)=1$ and $\lambda_{\mathrm{o}}(1)=0$. We also assume that these functions are such that $0<\lambda_{*} \leq \lambda_{T}(s) \leq 1$ for all $s \in[0,1]$. An illustrative example is $\lambda_{\mathrm{w}}(s)=s^{2}$ and $\lambda_{\mathrm{o}}(s)=(1-s)^{2}$. With these assumptions (1.6) is an elliptic equation.

From Darcy's law for each phase we get

$$
\begin{aligned}
& \lambda_{\mathrm{w}} \boldsymbol{v}_{\mathrm{o}}=-\lambda_{\mathrm{w}} \lambda_{\mathrm{o}} \nabla_{\boldsymbol{x}} p_{\mathrm{o}}+\lambda_{\mathrm{w}} \lambda_{\mathrm{o}} \rho_{\mathrm{o}} G \\
& \lambda_{\mathrm{o}} \boldsymbol{v}_{\mathrm{w}}=-\lambda_{\mathrm{w}} \lambda_{\mathrm{o}} \nabla_{\boldsymbol{x}} p_{\mathrm{w}}+\lambda_{\mathrm{w}} \lambda_{\mathrm{\circ}} \rho_{\mathrm{w}} G .
\end{aligned}
$$

Subtracting the second of these from the first

$$
\begin{aligned}
\lambda_{\mathrm{w}} \lambda_{\mathrm{o}} \nabla_{\boldsymbol{x}} p_{\mathrm{cow}} & =-\lambda_{\mathrm{w}} \boldsymbol{v}_{\mathrm{o}}+\lambda_{\mathrm{o}} \boldsymbol{v}_{\mathrm{w}}+\lambda_{\mathrm{w}} \lambda_{\mathrm{o}}\left(\rho_{\mathrm{o}}-\rho_{\mathrm{w}}\right) G \\
& =\lambda_{T} \boldsymbol{v}_{\mathrm{w}}-\lambda_{\mathrm{w}} \boldsymbol{v}_{T}+\lambda_{\mathrm{w}} \lambda_{\mathrm{o}}\left(\rho_{\mathrm{o}}-\rho_{\mathrm{w}}\right) G .
\end{aligned}
$$

We can solve for $\boldsymbol{v}_{\mathrm{w}}$

$$
\boldsymbol{v}_{\mathrm{w}}=f(s) \boldsymbol{v}_{T}+\frac{\lambda_{\mathrm{w}}(s) \lambda_{\mathrm{o}}(s)}{\lambda_{T}(s)}\left(\rho_{\mathrm{w}}-\rho_{\mathrm{o}}\right) G+\frac{\lambda_{\mathrm{w}}(s) \lambda_{\mathrm{o}}(s)}{\lambda_{T}(s)} \nabla_{\boldsymbol{x}} p_{\mathrm{cow}}(s) .
$$

Rewriting the continuity equation (1.2) for $s$ we find that

$$
s_{t}+\operatorname{div}_{\boldsymbol{x}}\left[f(s) \boldsymbol{v}_{T}+\lambda_{\mathrm{o}}(s) f(s)\left(\rho_{\mathrm{w}}-\rho_{\mathrm{o}}\right) G\right]=\operatorname{div}_{\boldsymbol{x}}\left[\lambda_{\mathrm{o}}(s) f(s) \nabla_{\boldsymbol{x}} p_{\text {cap }}(s)\right],
$$


where $p_{\text {cap }}=-p_{\text {cow }}$. It is assumed that the mapping $s \mapsto p_{\text {cap }}(s)$ is non-decreasing. Furthermore, the right hand side of (1.7) vanishes when $s=1$ or $s=0$. This equation is therefore a degenerate convection-diffusion equation. If we define

$$
a(s)=\lambda_{\mathrm{o}}(s) f(s) p_{\text {cap }}^{\prime}(s) \text { and } A(s)=\int_{0}^{s} a(\sigma) d \sigma
$$

then the standard Darcy model for two phase flow in an isotropic homogeneous porous medium reads

$$
\begin{gathered}
\operatorname{div}_{\boldsymbol{x}} \boldsymbol{v}_{T}=0, \quad \boldsymbol{v}_{T}=-\lambda_{T}(s) \nabla_{\boldsymbol{x}} p+\left(\lambda_{\mathrm{w}}(s) \rho_{\mathrm{w}}+\lambda_{\mathrm{o}}(s) \rho_{\mathrm{o}}\right) G, \\
s_{t}+\operatorname{div}_{\boldsymbol{x}}\left[f(s) \boldsymbol{v}_{T}+\lambda_{\mathrm{o}}(s) f(s)\left(\rho_{\mathrm{w}}-\rho_{\mathrm{o}}\right) G\right]=\Delta A(s),
\end{gathered}
$$

with $\Delta$ denoting $\operatorname{div}_{\boldsymbol{x}} \nabla_{\boldsymbol{x}}$. These two equations are often referred to as the pressure equation and the saturation equation respectively. Of course, (1.8) is supposed to hold in some domain $\Omega \subset \mathbb{R}^{d}$, and one must prescribe initial and boundary values. Since $A^{\prime}(s)$ vanishes for some $s$, one can expect discontinuities or sharp gradients in $s$ to form, even for smooth initial data. Therefore (1.8) must be considered in the weak, or rather entropy, sense. Due to the expected low regularity of $s$, one cannot expect that $p$ is regular, say $H^{1}$. Therefore the total velocity field $\boldsymbol{v}_{T}$ will probably not be sufficiently regular so that weak solutions can be easily defined.

Due to such difficulties, the problem of proving well-posedness of global weak solution to (1.8) has remained open for many decades. The total velocity $\boldsymbol{v}_{T}$ plays the role of a coefficient in the saturation equation. Although conservation laws, and degenerate convection diffusion equations, with space dependent coefficients have been extensively studied, see $[2,3,6,8,10,12,14,15]$ and references therein, the best results to this date require that the coefficient is of bounded variation. There have been many attempts to prove that $\boldsymbol{v}_{T}$ is a $B V$ function, or lies in some Sobolev space, but none of these have been successful. Partial results, with strong assumptions on the solution or the velocity field have been proved in [19, 21].

Due to these difficulties with the standard model (1.8), it is tempting to consider alternative models, or modifications of (1.8). In many situations, the capillary pressure $p_{\text {cow }}$ is quite small compared with other quantities in (1.8), as a consequence the diffusion $A$ is ignored, in which case the saturation equation is a scalar conservation law with a (possibly) irregular coefficient $\boldsymbol{v}_{T}$. The usual Kruzkov entropy condition for scalar conservation laws is chosen by requiring that the entropy solution is the limit of vanishing viscosity solutions, i.e., the limit as $\varepsilon \downarrow 0$ of solutions of (1.8) with the right hand side of the saturation equation replaced by $\varepsilon \Delta s$. The viscous regularization of the standard Darcy model reads

$$
\begin{gathered}
\operatorname{div}_{\boldsymbol{x}} \boldsymbol{v}_{T}^{\varepsilon}=0, \quad \boldsymbol{v}_{T}^{\varepsilon}=-\lambda_{T}\left(s^{\varepsilon}\right) \nabla_{\boldsymbol{x}} p^{\varepsilon}+\left(\lambda_{\mathrm{w}}\left(s^{\varepsilon}\right) \rho_{\mathrm{w}}+\lambda_{\mathrm{o}}\left(s^{\varepsilon}\right) \rho_{\mathrm{o}}\right) G, \\
s_{t}^{\varepsilon}+\operatorname{div}_{\boldsymbol{x}}\left[f\left(s^{\varepsilon}\right) \boldsymbol{v}_{T}^{\varepsilon}+\lambda_{\mathrm{o}}\left(s^{\varepsilon}\right) f\left(s^{\varepsilon}\right)\left(\rho_{\mathrm{w}}-\rho_{\mathrm{o}}\right) G\right]=\varepsilon \Delta s^{\varepsilon} .
\end{gathered}
$$

Due to the regularizing effect of $\Delta s$, this system of equations is well-posed, see $[17,16,11]$. Although it has not been proved that $s^{\varepsilon} \rightarrow s$ as $\varepsilon \downarrow 0$, it is generally believed that for small $\varepsilon, s^{\varepsilon}$ is close to a solution of (1.8) with $A \equiv 0$.

Observe that the presence of a degenerate diffusion term does not necessarily make the standard models more amenable to analysis, for clarity and brevity of exposition, therefore we shall assume that the capillary pressure is zero in the remainder of this paper.

1.2. The Brinkman model. The difficulties in obtaining well-posedness of the standard model raise a fundamental question - Is Darcy's law correct? In 1949, Brinkman [5] suggested the following modification of Darcy's law

$$
-\mu \Delta \boldsymbol{v}_{i}+\boldsymbol{v}_{i}=-\lambda_{i}(s) \nabla_{\boldsymbol{x}} p+\lambda_{i}(s) \rho_{i} G, \text { for } i=\mathrm{o}, \mathrm{w},
$$


where $\mu$ is a (small) positive constant, which we for simplicity assume is the same for both phases, and $p$ denotes the (common to the two phases) pressure. In [5] (1.10) was put forward for a single phase, but it has also been used in the two-phase setting [4].

Adding the two velocities, and applying the divergence operator to the result yields

$$
0=\operatorname{div}_{\boldsymbol{x}}\left[\lambda_{T}(s) \nabla_{\boldsymbol{x}} p-\left(\lambda_{\mathrm{o}}(s) \rho_{\mathrm{o}}+\lambda_{\mathrm{w}}(s) \rho_{\mathrm{w}}\right) G\right] .
$$

With Brinkman's law replacing Darcy's, the pressure $p^{\mu}$ and the water saturation $s^{\mu}$ satisfy

$$
\begin{aligned}
\operatorname{div}_{\boldsymbol{x}} \boldsymbol{v}_{T}^{\mu}=0, \quad \boldsymbol{v}_{T}^{\mu} & =-\lambda_{T}\left(s^{\mu}\right) \nabla_{\boldsymbol{x}} p^{\mu}+\left(\lambda_{\mathrm{o}}\left(s^{\mu}\right) \rho_{\mathrm{o}}+\lambda_{\mathrm{w}}\left(s^{\mu}\right) \rho_{\mathrm{w}}\right) G, \\
-\mu \Delta \boldsymbol{v}_{\mathrm{w}}^{\mu}+\boldsymbol{v}_{\mathrm{w}}^{\mu} & =-\lambda_{\mathrm{w}}\left(s^{\mu}\right) \nabla_{\boldsymbol{x}} p^{\mu}+\lambda_{\mathrm{w}}\left(s^{\mu}\right) \rho_{\mathrm{w}} G, \\
s_{t}^{\mu}+\operatorname{div}_{\boldsymbol{x}} \boldsymbol{v}_{\mathrm{w}}^{\mu} & =0 .
\end{aligned}
$$

In order to be well-posed, also this system must be coupled with initial and boundary conditions. In contrast to the standard model, and due to the regularizing effect of the Helmholtz operator $-\mu \Delta+\mathrm{Id}$, the saturation $s^{\mu}$ is differentiable and continuous for $\mu>0$. The limit of the system (1.11) as $\mu$ vanishes is (1.8), never the less, we shall see that the limits of the corresponding solutions are quite different.

1.3. Helmholtz reguarization. Now we shall present yet another model, the basis for which is the assumption that on a scale much smaller than the pores themselves, the two phases "see each other" much like a single phase sees the pores. In order to motivate this, we first recall the standard motivation for Darcy's law for one phase flow [20].

Derivation of Darcy's law for one phase flow. Assume that we have an incompressible single phase flow in a porous medium, with a velocity $\boldsymbol{v}$. Assume furthermore that $v$ satisfies Stokes equation (potential flow)

$$
\Delta \boldsymbol{v}=\nabla_{\boldsymbol{x}} p .
$$

The continuity equation $\operatorname{div}_{\boldsymbol{x}} \boldsymbol{v}=0$ then implies that

$$
\Delta p(\boldsymbol{x})=0 .
$$

for $\boldsymbol{x}$ in the porous space. This porous space consists of innumerable twisty channels, confined by an outer boundary $\partial \Omega$, where $\Omega$ is the domain of interest. Let $\Omega_{\phi}$ denote the porous space, so that

$$
\Omega=\Omega_{\phi} \cup\{\text { the impermeable part of } \Omega .\}
$$

Let $\chi_{\phi}$ denote the characteristic function of the porous space, and let $N$ be some averaging kernel. Then define the porosity

$$
\phi(\boldsymbol{x})=\int_{\Omega} N(\boldsymbol{x}-\boldsymbol{y}) \chi_{\phi}(\boldsymbol{y}) d \boldsymbol{y} .
$$

We assume that the porous medium is homogenenous, hence $\phi$ is constant. For a quantity $f=f(x)$ we define the averages

$$
\langle f\rangle=\int_{\Omega} N(\boldsymbol{x}-\boldsymbol{y}) \chi_{\phi}(\boldsymbol{y}) f(\boldsymbol{y}) d \boldsymbol{y} .
$$

Next we have the relation

$$
\begin{aligned}
\left\langle\nabla_{\boldsymbol{x}} f\right\rangle(\boldsymbol{x}) & =\iint_{\Omega \cap \Omega_{\phi}} N(\boldsymbol{x}-\boldsymbol{y}) \nabla_{\boldsymbol{y}} f(\boldsymbol{y}) d \boldsymbol{y} \\
& =\nabla_{\boldsymbol{x}}\langle f\rangle(\boldsymbol{x})+\int_{\partial \Omega_{\phi}} N(\boldsymbol{x}-\boldsymbol{y}) f(\boldsymbol{y}) \boldsymbol{n}(\boldsymbol{y}) d S(\boldsymbol{y}),
\end{aligned}
$$


where $\boldsymbol{n}(\boldsymbol{y})$ denotes the unit normal to $\partial \Omega_{\phi}$ at a point $\boldsymbol{y}$. Since $p$ is harmonic in $\Omega \cap \Omega_{\phi}$, see (1.12), then we have

$$
p(\boldsymbol{x})=\int_{\partial\left(\Omega \cap \Omega_{\phi}\right)} p_{\mathrm{b}}(\boldsymbol{y}) \nabla_{\boldsymbol{x}} H(\boldsymbol{x}-\boldsymbol{y}) \cdot \boldsymbol{n}(\boldsymbol{y}) d S(\boldsymbol{y}),
$$

where $H$ is the Green's function on $\Omega \cap \Omega_{\phi}$ with $p_{\mathrm{b}}$ the imposed boundary value. Observe that $p$, and hence also $\boldsymbol{v}$, are linearly dependent on the boundary values. Hence we can apply a scaling argument as in [20] to show that

$$
\boldsymbol{v}=A\langle\boldsymbol{v}\rangle
$$

for some symmetric matrix $A$ which is independent of the boundary conditions. Applying the Laplacian and the averaging operator $\langle\cdot\rangle$ to this, we obtain

$$
\left\langle\nabla_{\boldsymbol{x}} p\right\rangle=\langle\Delta \boldsymbol{v}\rangle=\langle\Delta(A\langle\boldsymbol{v}\rangle)\rangle \approx\langle\Delta A\rangle\langle\boldsymbol{v}\rangle,
$$

or

$$
\nabla_{\boldsymbol{x}}\langle p\rangle+\int_{\partial \Omega_{\phi}} N(\boldsymbol{x}-\boldsymbol{y}) p(\boldsymbol{y}) \boldsymbol{n}(\boldsymbol{y}) d S(\boldsymbol{y}) \approx\langle\Delta A\rangle\langle\boldsymbol{v}\rangle .
$$

It turns out that the matrix $\langle\Delta A\rangle$ is invertible, we call its inverse, $K$, the permeability. Furthermore, it is often reasonable to assume that the porous channels are so twisty that the integral along their boundary vanishes. In this case we are left with Darcy's law for one phase flow

$$
\langle\boldsymbol{v}\rangle=K \nabla_{\boldsymbol{x}}\langle p\rangle .
$$

Motivation of the Helmholtz model. In order to motivate an alternative to Darcy's law for two phase flow we can argue as follows. Assume that we have two phases, oil and water, these are chemically inert and do not dissolve. However on the scale of the pores, they are sufficiently mixed so that we can define the saturation. In Figure 1 this is called the "saturation scale". On a very small scale, much smaller than that of the pores, one phase will act similarly to the way the porous walls act on the fluid for single phase flow. This is called the "two phase scale" in Figure 1. On the saturation scale we therefore postulate two Darcy's laws

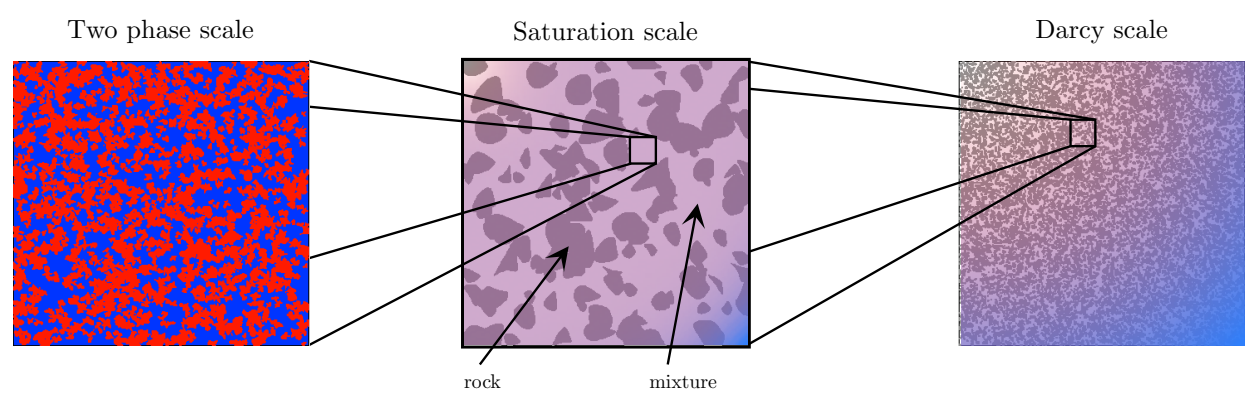

Figure 1. The three scales.

$$
\left\langle\boldsymbol{v}_{i}\right\rangle_{N}=K_{i} \nabla_{\boldsymbol{x}}\left\langle p_{i}\right\rangle_{N}, \quad i=\mathrm{o}, \mathrm{w},
$$

where we have indicated the dependence on the averaging kernel $N$. If the two fluids are uniformly mixed in each direction, then $K_{i}=\lambda_{i} I$. The coefficient $\lambda_{i}$ will be a function of the saturation. The continuity equations for each phase then read

$$
\operatorname{div}_{\boldsymbol{x}}\left[\lambda_{i} \nabla_{\boldsymbol{x}}\left\langle p_{i}\right\rangle_{N}\right]=0, \boldsymbol{x} \in \Omega_{\phi},
$$

with boundary conditions

$$
\begin{cases}\lambda_{i} \nabla_{\boldsymbol{x}}\left\langle p_{i}\right\rangle_{N}=0, & \boldsymbol{x} \in \partial \Omega_{\phi}, \\ \lambda_{i} \nabla_{\boldsymbol{x}}\left\langle p_{i}\right\rangle_{N}=f_{i} & \boldsymbol{x} \in \partial \Omega \cap \Omega_{\phi} .\end{cases}
$$


The phase velocities are linearly dependent on these boundary conditions, so scale arguments lead to the relation

$$
\left\langle\boldsymbol{v}_{i}\right\rangle_{N}=A_{i}\left\langle\left\langle\boldsymbol{v}_{i}\right\rangle_{N}\right\rangle_{M}, \quad i=\mathrm{o}, \mathrm{w},
$$

where $M$ is (another) averaging kernel. We now assume that the porous walls do not discriminate between the two phases, this means that $A_{\mathrm{w}}=A_{\mathrm{o}}=A$. Then we have

$$
\begin{aligned}
\left\langle\left\langle\boldsymbol{v}_{i}\right\rangle_{N}\right\rangle_{M} & =\left\langle A^{-1} \lambda_{i} \nabla_{\boldsymbol{x}}\left\langle p_{i}\right\rangle_{N}\right\rangle_{M} \\
& \approx\left\langle A^{-1} \lambda_{i}\right\rangle_{M}\left\langle\nabla_{\boldsymbol{x}}\left\langle p_{i}\right\rangle_{N}\right\rangle_{M} \\
& =\left\langle A^{-1} \lambda_{i}\right\rangle_{M} \nabla_{\boldsymbol{x}}\left\langle\left\langle p_{i}\right\rangle_{N}\right\rangle_{M}+\left\langle A^{-1} \lambda_{i}\right\rangle_{M} \int_{\partial \Omega_{\phi}}\left\langle p_{i}\right\rangle_{N}(y) M(x-y) \boldsymbol{n}(y) d S(y) .
\end{aligned}
$$

We assume that the above integral is zero due to the mixing of the phases. We also assume that the saturation changes rapidly compared with the size of the support of $M$. We are interested in $\tilde{p}_{i}=\left\langle\left\langle p_{i}\right\rangle_{N}\right\rangle_{M}$. The continuity equation then implies

$$
\operatorname{div}_{\boldsymbol{x}}\left(\left\langle A^{-1} \lambda_{i}\right\rangle_{M} \nabla_{\boldsymbol{x}} \tilde{p}_{i}\right)=0 .
$$

Let us for simplicity set $A=I$, and choose $M$ to be the Helmholtz kernel $(-\eta \Delta+$ Id $)^{-1}$. We define $\Lambda_{i}=(-\eta \Delta+I)^{-1} \lambda_{i}$, or

$$
-\eta \Delta \Lambda_{i}+\Lambda_{i}=\lambda_{i}
$$

- We then end up with the usual saturation equation, but the pressure is more regular than usual.

Concretely, the "Helmholtz" model for two phase flow in porous media reads

$$
\begin{aligned}
& \left\{\begin{array}{l}
\operatorname{div}_{\boldsymbol{x}} \boldsymbol{v}_{T}^{\eta}=0, \quad-\boldsymbol{v}_{T}^{\eta}=-\Lambda_{T} \nabla_{\boldsymbol{x}} p^{\eta}+\left(\Lambda_{\mathrm{o}} \rho_{\mathrm{o}}+\Lambda_{\mathrm{w}} \rho_{\mathrm{w}}\right) G, \\
-\eta \Delta \Lambda_{i}+\Lambda_{i}=\lambda_{i}\left(s^{\eta}\right), \quad i=\mathrm{o}, \mathrm{w}, \\
\Lambda_{T}=\Lambda_{\mathrm{o}}+\Lambda_{\mathrm{w}},
\end{array}\right. \\
& s_{t}^{\eta}+\operatorname{div}_{\boldsymbol{x}}\left(f\left(s^{\eta}\right) \boldsymbol{v}_{T}^{\eta}+\left(\lambda_{\mathrm{o}}\left(s^{\eta}\right) f\left(s^{\eta}\right)\left(\rho_{\mathrm{w}}-\rho_{\mathrm{o}}\right)\right) G\right)=0 .
\end{aligned}
$$

The first three lines above constitute the pressure equation, and the last is called the saturation equation. For this model, the coefficient in the saturation equation, $\boldsymbol{v}_{T}^{\eta}$ is smooth, entropy solutions are easily defined for $\eta>0$. As in the standard model, discontinuities in the saturation will typically occur. Hence, if one thinks that it is important for models to capture discontinuities in $s$, one may prefer the Helmholtz model over the viscous regularization (1.9) or the Brinkman model (1.11). The Helmholtz regularization was first introduced and analyzed in [7]. Observe that formally, (1.13) reduces to the inviscid standard model (2.1) as $\eta \rightarrow 0$.

Remark. It is worth looking at what these models are in one spatial dimension. In this case the continuity equation $0=\operatorname{div}_{\boldsymbol{x}} \boldsymbol{v}=\boldsymbol{v}_{x}$ implies that $\boldsymbol{v}=$ constant. If we use units so that this constant is one, and assume that the densities are equal, the three different models read

$$
\begin{aligned}
s_{t}^{\varepsilon}+f\left(s^{\varepsilon}\right)_{x} & =\varepsilon s_{x x}^{\varepsilon}, \quad \text { viscous regularization, } \\
s_{t}^{\mu}-\mu s_{x x t}^{\mu}+f\left(s^{\mu}\right)_{x} & =0, \text { the Brinkman model, } \\
s_{t}^{\eta}+f\left(s^{\eta}\right)_{x} & =0, \text { the Helmholtz model. }
\end{aligned}
$$

We see that the Helmholtz model is independent of $\eta$, and indeed constitutes a scalar conservation law whose entropy solutions are the limit of $s^{\varepsilon}$ as $\varepsilon \downarrow 0$. As shown in [9], the limits as $\mu \downarrow 0$ of $s^{\mu}$ can contain so-called "non-classical" shocks, see [18] for a definition of this. Thus for small $\mu, s^{\mu}$ is not necessarily close to $s^{\eta}$. 


\section{NUMERICAL EXPERIMENTS}

In this section we study two phase flow in a two (space) dimensional setting. The inviscid variant of (1.9) then reads

$$
\begin{gathered}
\operatorname{div}_{\boldsymbol{x}} \boldsymbol{v}_{T}=0, \quad \boldsymbol{v}_{T}=-\lambda_{T}(s) \nabla_{\boldsymbol{x}} p+\left(\lambda_{\mathrm{w}}(s) \rho_{\mathrm{w}}+\lambda_{\mathrm{o}}(s) \rho_{\mathrm{o}}\right) G, \\
s_{t}+\operatorname{div}_{\boldsymbol{x}}\left[f(s) \boldsymbol{v}_{T}+\lambda_{\mathrm{o}}(s) f(s)\left(\rho_{\mathrm{w}}-\rho_{\mathrm{o}}\right) G\right]=0 .
\end{gathered}
$$

This is supposed to hold for $t>0$ and for $x$ in some domain $\Omega \subset \mathbb{R}^{2}$, and supplemented with initial and boundary conditions.

In several space dimensions the convergence as $\mu \downarrow 0$ of solutions to the Helmholtz model to solutions of (2.1) is not known. From the examples in [9] we know that solutions of the Brinkman model will not always converge to solutions of (2.1). As mentioned before, it is generally believed that solutions of (1.9) converge to solutions of (2.1) as $\varepsilon \downarrow 0$, and this convergence has also been studied extensively for numerical approximations. In this section we shall therefore compare numerical solutions to (2.1) with numerical solutions to (1.11) and (1.13) in two dimensions and for different values of $\mu$ and $\eta$.

2.1. A quarter five spot. The "quarter five spot" setup is commonly used is reservoir simulation. Here we shall use it to simulate injection of water into a porous medium filled with mainly oil. The setting is horizontal $(G=0)$ and periodic in the plane, with "injections wells" are centered at lattice points in $\mathbb{N}^{2}$ with both coordinates even, and "production wells" at lattice points with both coordinates odd. An injection well situated at $(k, l)$ is described by a source term $q_{k l}=\tilde{q}(r)$, where $r$ is the distance from $(k, l)$. We also assume that the production well situated at $(k+1, l+1)$ is described by the source term $-q_{k l}$. In this case we can reduce the setting to the domain $\Omega=(0,1)^{2}$ and the inviscid model reads

$$
\begin{aligned}
& \operatorname{div}_{\boldsymbol{x}}\left(\lambda_{T}(s) \nabla_{\boldsymbol{x}} p\right)=q, \\
& \left.\begin{array}{rl}
\boldsymbol{v}_{T} & =-\lambda_{T}(s) \nabla_{\boldsymbol{x}} p, \\
s_{t}+\operatorname{div}_{\boldsymbol{x}}\left[\boldsymbol{v}_{T} f(s)\right] & =\max \{q, 0\}-f(s) \min \{q, 0\},
\end{array}\right\} t>0, \quad \boldsymbol{x} \in \Omega, \\
& \left.\begin{array}{l}
\nabla_{\boldsymbol{x}} p \cdot \boldsymbol{n}=0 \\
\nabla_{\boldsymbol{x}} s \cdot \boldsymbol{n}=0
\end{array}\right\}, \quad t>0, \quad \boldsymbol{x} \in \partial \Omega, \\
& s(0, x)=s_{0}(x), \quad \boldsymbol{x} \in \Omega,
\end{aligned}
$$

where $q=q_{0,0}-q_{1,1}$ and $\boldsymbol{n}$ is a unit normal to $\partial \Omega$. The pressure is normalized so that $\iint_{\Omega} p d x=0$. For the source term, we choose

$$
\tilde{q}(r)=C \begin{cases}1 & r<\sigma, \\ \frac{1}{2}\left(\cos \left(\pi \frac{r-\sigma}{\sigma}\right)+1\right) & \sigma \leq r<2 \sigma, \quad \sigma=\frac{\sqrt{2}}{15} \\ 0 & 2 \sigma \leq r\end{cases}
$$

and $C$ is chosen such that $\iint \tilde{q} d \boldsymbol{x}=4$.

The Brinkman and the Helmholtz models for this situation read

$$
\begin{aligned}
& \operatorname{div}_{\boldsymbol{x}}\left(\lambda_{T}\left(s^{\mu}\right) \nabla_{\boldsymbol{x}} p^{\mu}\right)=q, \\
&-\mu \Delta \boldsymbol{v}_{\mathrm{w}}^{\mu}+\boldsymbol{v}_{\mathrm{w}}^{\mu}=-\lambda_{\mathrm{w}}\left(s^{\mu}\right) \nabla_{\boldsymbol{x}} p^{\mu} \\
& s_{t}^{\mu}+\operatorname{div}_{\boldsymbol{x}}\left[\boldsymbol{v}_{\mathrm{w}}^{\mu}\right]= \max \{q, 0\}-f\left(s^{\mu}\right) \min \{q, 0\}, \quad \\
& \nabla_{\boldsymbol{x}} p^{\mu} \cdot \boldsymbol{n}=0 \\
& \nabla_{\boldsymbol{x}} s^{\mu} \cdot \boldsymbol{n}=0 \\
& {\left[\nabla_{\boldsymbol{x}} \boldsymbol{v}_{\mathrm{w}}^{\mu}\right] \boldsymbol{n}=0 } \\
& s^{\mu}(0, x)=s_{0}(x), \quad \boldsymbol{x} \in \Omega, \quad \boldsymbol{x} \in \Omega, \quad \boldsymbol{x} \in \partial \Omega,
\end{aligned}
$$


and

$$
\begin{gathered}
-\eta \Delta \Lambda_{i}+\Lambda_{i}=\lambda_{i}\left(s^{\eta}\right), \quad i=\mathrm{o}, \mathrm{w}, \\
\operatorname{div}_{\boldsymbol{x}}\left(\Lambda_{T}\left(s^{\eta}\right) \nabla_{\boldsymbol{x}} p^{\eta}\right)=q, \\
\boldsymbol{v}_{T}^{\eta}=-\Lambda_{T}\left(s^{\eta}\right) \nabla_{\boldsymbol{x}} p^{\eta}, \Lambda_{T}=\Lambda_{\mathrm{o}}+\Lambda_{\mathrm{w}}, \\
s_{t}^{\eta}+\operatorname{div}_{\boldsymbol{x}}\left[\boldsymbol{v}_{T}^{\eta} f\left(s^{\eta}\right)\right]=\max \{q, 0\}-f\left(s^{\eta}\right) \min \{q, 0\}, \\
\nabla_{\boldsymbol{x}} p^{\eta} \cdot \boldsymbol{n}=0 \\
\nabla_{\boldsymbol{x}} s^{\eta} \cdot \boldsymbol{n}=0 \\
\nabla_{\boldsymbol{x}} \Lambda_{i} \cdot \boldsymbol{n}=0 \\
s^{\eta}(0, x)=s_{0}(x), \quad \boldsymbol{x} \in \Omega, \quad \boldsymbol{x} \in \Omega, \\
\end{gathered}
$$

respectively. The models (2.3) and (2.4) use the same normalization for the pressure, $\iint_{\Omega} p d \boldsymbol{x}=0$.

We remark that in the rest of this paper we use the following expression for the relative permeabilities

$$
\lambda_{\mathrm{w}}(s)=s^{2}, \quad \lambda_{\mathrm{o}}(s)=(1-s)^{2},
$$

and the notation $(x, y)=\boldsymbol{x}$.

To solve these equations numerically, we replace the derivatives with finite differences. Choose an integer $N \geq 2$, and set $\Delta x=\Delta y=1 /(N+1)$, set $x_{i}=$ $(i-1 / 2) \Delta x, y_{j}=(j-1 / 2) \Delta y$ for $i, j=1, \ldots, N$. We use the common notation that $u_{i j} \approx u\left(x_{i}, y_{j}\right)$ for any sought function $u$. We also introduce the discrete derivatives

$$
D_{x}^{ \pm} u_{i j}= \pm \frac{u_{i j}-u_{i \mp 1, j}}{\Delta x} \text {, and } D_{y}^{ \pm} u_{i j}= \pm \frac{u_{i j}-u_{i, j \mp 1}}{\Delta y}
$$

With this notation, the scheme for the boundary value problem

$$
-\varepsilon \Delta u+u=f, \quad x \in \Omega, \quad \nabla_{\boldsymbol{x}} u \cdot \boldsymbol{n}=0, \quad \boldsymbol{x} \in \partial \Omega,
$$

reads

$$
\begin{gathered}
-\varepsilon\left(D_{x}^{+} D_{x}^{-}+D_{y}^{+} D_{y}^{-}\right) u_{i j}+u_{i j}=f_{i j}, \quad i, j=1, \ldots, N, \\
D_{x}^{-} u_{1, j}=D_{x}^{+} u_{N, j}=D_{y}^{-} u_{i, 1}=D_{y}^{+} u_{i, N}=0 .
\end{gathered}
$$

We write the solution $u_{i j}=H^{\varepsilon}[f]_{i j}$. To solve the pressure equation

$$
\operatorname{div}_{\boldsymbol{x}}\left[l \nabla_{\boldsymbol{x}} p\right]=h \quad \boldsymbol{x} \in \Omega, \quad \nabla_{\boldsymbol{x}} p \cdot \boldsymbol{n}=0, \quad \boldsymbol{x} \in \partial \Omega, \quad \iint_{\Omega} p d \boldsymbol{x}=0
$$

we use the scheme

$$
\begin{gathered}
D_{x}^{-}\left[l_{i+1 / 2, j} D_{x}^{+} p_{i j}\right]+D_{y}^{-}\left[l_{i, j+1 / 2} D_{y}^{+} p_{i j}\right]=h_{i j}, \quad i, j=1, \ldots, N, \\
D_{x}^{-} p_{1, j}=D_{x}^{+} p_{N, j}=D_{y}^{-} p_{i, 1}=D_{y}^{+} p_{i, N}=0,
\end{gathered}
$$

with the extra condition $\sum_{i j} p_{i j}=0$, and where

$$
l_{i+1 / 2, j}=\frac{2 l_{i j} l_{i+1, j}}{l_{i j}+l_{i+1, j}},
$$

and $l_{i, j+1 / 2}$ is defined analogously. We write the solution $p_{i j}=P^{l}[h]_{i j}$. It remains to define a scheme for the conservation law.

$$
s_{t}+\operatorname{div}_{\boldsymbol{x}}[\boldsymbol{v} f(s)]=q, \quad \boldsymbol{x} \in \Omega, \quad \nabla_{\boldsymbol{x}} s \cdot \boldsymbol{n}=0, \quad \boldsymbol{x} \in \partial \Omega .
$$

To this end, set $\boldsymbol{v}=(u, v)$, and let $F=F(w ; s, \sigma)$ be a two point numerical flux, see [13], such that $F(w ; s, s)=w f(s)$, and $F$ is continuous and non-decreasing in $s$, and 
continuous non-increasing in $\sigma$. The (finite volume) scheme for the conservation law then reads

$$
\begin{gathered}
D_{+}^{t} s_{i j}^{n}+D_{x}^{-}\left[F\left(u_{i+1 / 2, j} ; s_{i j}^{n}, s_{i+1, j}^{n}\right)\right] \\
\quad+D_{y}^{-}\left[F\left(v_{i, j+1 / 2} ; s_{i j}^{n}, s_{i, j+1}^{n}\right)\right]=q_{i j}^{n} \quad i, j=1, \ldots, N, \\
D_{x}^{-} s_{1, j}^{n}=D_{x}^{+} s_{N, j}^{n}=D_{y}^{-} s_{i, 1}^{n}=D_{y}^{+} s_{i, N}^{n}=0, \quad s_{i j}^{0}=s\left(0, x_{i}, y_{j}\right) .
\end{gathered}
$$

where

$$
D_{+}^{t} s_{i j}^{n}=\frac{s_{i j}^{n+1}-s_{i j}^{n}}{\Delta t},
$$

for some small positive $\Delta t$. We write the solution $s^{n+1}=C^{\boldsymbol{v}}\left[s^{n}\right]$.

To solve the equation

$$
s_{t}+\operatorname{div}_{\boldsymbol{x}} \boldsymbol{v}=q, \quad \boldsymbol{x} \in \Omega, \quad \nabla_{\boldsymbol{x}} s \cdot \boldsymbol{n}=0, \quad \boldsymbol{x} \in \partial \Omega,
$$

we propose the simple scheme

$$
\begin{gathered}
D_{+}^{t} s_{i j}^{n}+D_{x}^{-} u_{i+1 / 2, j}+D_{y}^{-} v_{i, j+1 / 2}=q_{i j}, \quad i, j=1, \ldots, N, \\
D_{x}^{-} s_{1, j}^{n}=D_{x}^{+} s_{N, j}^{n}=D_{y}^{-} s_{i, 1}^{n}=D_{y}^{+} s_{i, N}^{n}=0, \quad s_{i j}^{0}=s\left(0, x_{i}, y_{j}\right),
\end{gathered}
$$

and we use the notation $s^{n+1}=T^{\boldsymbol{v}}\left[s^{n}\right]$.

The schemes (2.5), (2.6), (2.7) and (2.8) can be combined to yield schemes for (2.2), (2.3) and (2.4). The scheme for the standard Darcy model (2.2) is defined by repetition of the following steps:

Step 1: $\quad p=P^{\lambda_{T}\left(s^{n}\right)}\left[q^{n}\right]$,

Step 2: $\quad\left\{\begin{array}{l}u_{i+1 / 2, j}=\lambda_{T}\left(s^{n}\right)_{i+1 / 2, j} D_{x}^{+} p_{i j}, \\ v_{i, j+1 / 2}=\lambda_{T}\left(s^{n}\right)_{i, j+1 / 2} D_{y}^{+} p_{i j},\end{array}\right.$

Step 3: $\quad s^{n+1}=C^{(u, v)}\left[s^{n}\right]$.

Similarly, we propose the following scheme for (2.3):

$$
\begin{array}{ll}
\text { Step 1: } & p=P^{\lambda_{T}\left(s^{\mu, n}\right)}\left[q^{n}\right], \\
\text { Step 2: } & \boldsymbol{v}_{\mathrm{w}}=H^{\mu}\left[-\lambda_{\mathrm{w}}\left(s^{\mu, n}\right) \nabla_{\boldsymbol{x}} p\right], \\
\text { Step 3: } & s^{\mu, n+1}=T^{\boldsymbol{v}_{\mathrm{w}}\left[s^{\mu, n}\right],}
\end{array}
$$

and for $(2.4)$

$$
\begin{array}{ll}
\text { Step 1: } & \Lambda_{i}=H^{\eta}\left[\lambda_{i}\left(s^{\eta, n}\right)\right], \quad i=\mathrm{o}, \mathrm{w}, \\
\text { Step 2: } & p=P^{\Lambda_{T}}\left[q^{n}\right], \\
\text { Step 3: } & \left\{\begin{array}{l}
u_{i+1 / 2, j}=\left(\Lambda_{T}\right)_{i+1 / 2, j} D_{x}^{+} p_{i j}, \\
v_{i, j+1 / 2}=\left(\Lambda_{T}\right)_{i, j+1 / 2} D_{y}^{+} p_{i j},
\end{array}\right. \\
\text { Step 4: } & s^{n+1}=C^{(u, v)}\left[s^{n}\right] .
\end{array}
$$

In all cases, we assume that $\Delta x$ and $\Delta t$ are related via a so-called CFL-condition $\Delta t=c \Delta x$. For sufficiently small $c$, we can use similar arguments to those put forward in [9] and [7], to show that as $\Delta x$ to zero, the approximate solutions generated by the schemes (2.10) and (2.11) converge to weak solutions of (2.3) and (2.4) respectively. This holds for $\mu$ and $\eta$ strictly positive. There is presently no proof showing that approximate solutions generated by the algorithm (2.9) converge.

Here, we are interested in determining, via numerical experiments, how close the approximations generated by the three schemes $(2.9),(2.10)$ and $(2.11)$ are for 
small $\mu$ and $\eta$. From now on we use $\mu=\eta=\varepsilon$. As initial data we set

$$
s_{0}(x, y)= \begin{cases}1 & r<\sigma, \\ \frac{1}{2}\left(\cos \left(\pi \frac{r-\sigma}{\sigma}\right)+1\right) & \sigma \leq r<2 \sigma, \quad \sigma=\frac{\sqrt{2}}{15} . \\ 0 & 2 \sigma \leq r,\end{cases}
$$

In Figure 2 we show approximate solutions for $t=0.5$ for the three schemes, with $\varepsilon=0.1$ and $\Delta x=1 / 101$. We observe that the Darcy model and the Helmholtz
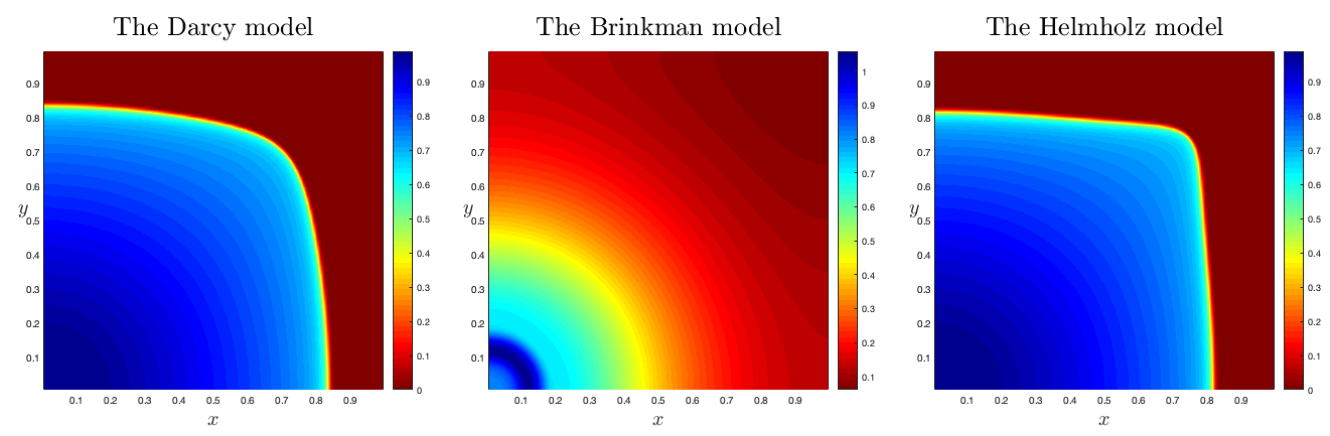

FiguRE 2. $\varepsilon=0.1$, left: (2.9), middle: (2.10), right: (2.11)

model give quite similar results, and that the water saturation computed by the Brinkman model is quite different. To investigate this further we compute the approximates solutions using $\Delta x=1 / 251$ for $\varepsilon=0.1, \varepsilon=0.005$ and $\varepsilon=0.0001$ for the Brinkman and the Helmholtz models. Figure 3 shows the diagonal of the saturation $\left(s_{i i}^{n}\right)$ at $t=0.5$. We see that the Helmholtz approximation seems to
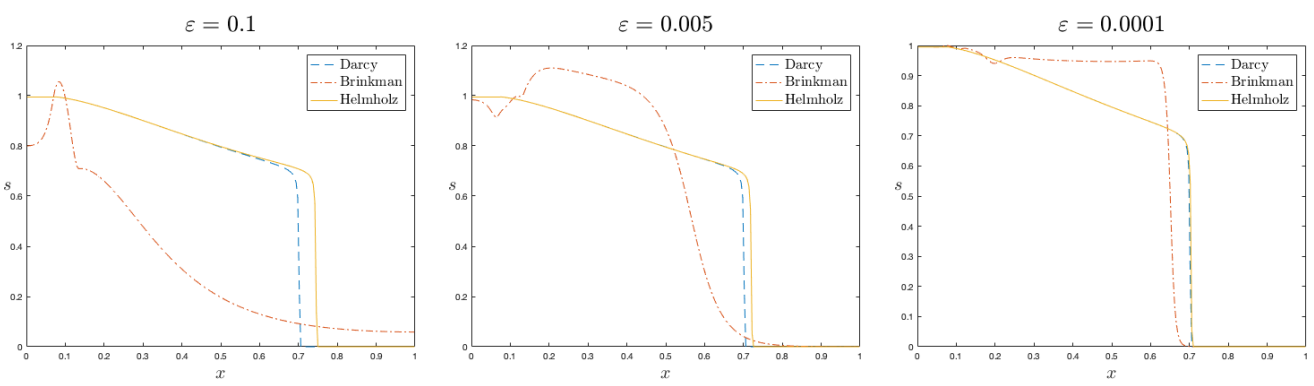

FiguRE 3. Saturation on the diagonal, left: $\varepsilon=0.1$, middle: $\varepsilon=$ 0.005, right: $\varepsilon=0.0001$.

converge to the Darcy approximation, whereas the Brinkman approximation seems to converge to a non-classical shock. This is in agreement with the one-dimensional numerical results reported in [9].

2.2. Gravity driven flow. Next we consider a situation where the dynamics are driven solely by gravity. The initial data consists of a heap of water in a vertical, two dimensional, homogeneous porous medium otherwise filled with oil. The upper and the lower boundary are impermeable, and the right so that after some (?) time, the all the water will be situated along the bottom of the domain.

To model this we use the domain $\Omega=(0,1 / 2) \times(0,1)$, and initial data

$$
s_{0}(x, y)= \begin{cases}1 & r<\sigma \\ \frac{1}{2}\left(\cos \left(\pi \frac{r-\sigma}{\sigma}\right)+1\right) & \sigma \leq r<2 \sigma \\ 0 & 2 \sigma \leq r\end{cases}
$$


where $\sigma=0.24$ and $r=\sqrt{(x-1 / 2)^{2}+(y-1 / 2)^{2}}$. We assume that the domain is vertical in the $y$-direction, and let

$$
\boldsymbol{G}=(0,1) \text { and } \rho_{\mathrm{o}}=1, \quad \rho_{\mathrm{w}}=4 .
$$

The standard Darcy model in this case reads

$$
\begin{gathered}
-\operatorname{div}_{\boldsymbol{x}}\left[\lambda_{T}(s) \nabla_{\boldsymbol{x}} p\right]=\frac{\partial}{\partial y}\left[\lambda_{\mathrm{o}}(s) \rho_{\mathrm{o}}+\lambda_{\mathrm{w}}(s) \rho_{\mathrm{w}}\right], \\
\boldsymbol{v}_{T}=-\lambda_{T}(s) \nabla_{\boldsymbol{x}} p+\left(\lambda_{\mathrm{w}}(s) \rho_{\mathrm{w}}+\lambda_{\mathrm{o}}(s) \rho_{\mathrm{o}}\right) \boldsymbol{G}, \quad \operatorname{si} \\
s_{t}+\operatorname{div}_{\boldsymbol{x}}\left[\boldsymbol{v}_{T} f(s)\right]+\frac{\partial}{\partial y}\left[\lambda_{\mathrm{o}}(s) f(s)\left(\rho_{\mathrm{w}}-\rho_{\mathrm{o}}\right)\right]=0, \quad \boldsymbol{x} \in \Omega \\
\boldsymbol{v}_{T} \cdot \boldsymbol{n}=0, \boldsymbol{v}_{T} \cdot \boldsymbol{\nu}=0, \\
\nabla_{\boldsymbol{x}} s \cdot \boldsymbol{n}=0, \\
s(0, \boldsymbol{x})=s_{0}(\boldsymbol{x}), \quad \boldsymbol{x} \in \Omega, \\
t>0, \boldsymbol{x} \in \partial \Omega,
\end{gathered}
$$

where $\boldsymbol{\nu}$ is a unit vector tangential to $\partial \Omega$. Similarly, the Brinkman and the Helmholtz models with $\mu=\eta=\varepsilon$ read

$$
\begin{aligned}
& \begin{aligned}
-\operatorname{div}_{\boldsymbol{x}}\left[\lambda_{T}\left(s^{\varepsilon}\right) \nabla_{\boldsymbol{x}} p\right] & =\frac{\partial}{\partial y}\left[\lambda_{\mathrm{o}}\left(s^{\varepsilon}\right) \rho_{\mathrm{o}}+\lambda_{\mathrm{w}}\left(s^{\varepsilon}\right) \rho_{\mathrm{w}}\right], \\
\boldsymbol{v}_{T}^{\varepsilon}=- & \lambda_{T}\left(s^{\varepsilon}\right) \nabla_{\boldsymbol{x}} p+\left(\lambda_{\mathrm{w}}\left(s^{\varepsilon}\right) \rho_{\mathrm{w}}+\lambda_{\mathrm{o}}\left(s^{\varepsilon}\right) \rho_{\mathrm{o}}\right) \boldsymbol{G}, \\
-\varepsilon \Delta \boldsymbol{v}_{\mathrm{w}}^{\varepsilon}+\boldsymbol{v}_{\mathrm{w}}^{\varepsilon} & =-\lambda_{\mathrm{w}}\left(s^{\varepsilon}\right) \nabla_{\boldsymbol{x}} p+\lambda_{\mathrm{w}}\left(s^{\varepsilon}\right) \boldsymbol{G}, \\
s_{t}^{\varepsilon}+\operatorname{div}_{\boldsymbol{x}}\left[\boldsymbol{v}_{\mathrm{w}}^{\varepsilon}\right] & =0,
\end{aligned} \quad t>0, \quad \boldsymbol{x} \in \Omega
\end{aligned}
$$

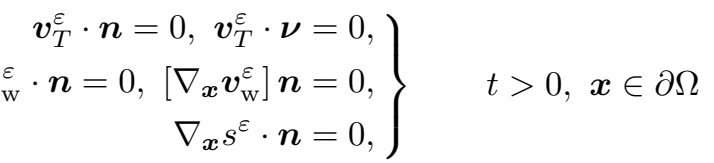

$$
\begin{aligned}
& s(0, \boldsymbol{x})=s_{0}(\boldsymbol{x}), \quad \boldsymbol{x} \in \Omega,
\end{aligned}
$$

and

$$
\begin{gathered}
-\varepsilon \Delta \Lambda_{i}^{\varepsilon}+\Lambda_{i}^{\varepsilon}=\lambda_{i}\left(s^{\varepsilon}\right), \quad i=\mathrm{o}, \mathrm{w}, \\
-\operatorname{div}_{\boldsymbol{x}}\left[\Lambda_{T}^{\varepsilon} \nabla_{\boldsymbol{x}} p\right]=\frac{\partial}{\partial y}\left[\Lambda_{\mathrm{o}}^{\varepsilon} \rho_{\mathrm{o}}+\Lambda_{\mathrm{w}}^{\varepsilon} \rho_{\mathrm{w}}\right], \\
\boldsymbol{v}_{T}^{\varepsilon}=-\Lambda_{T} \nabla_{\boldsymbol{x}} p+\left(\Lambda_{\mathrm{w}} \rho_{\mathrm{w}}+\Lambda_{\mathrm{o}} \rho_{\mathrm{o}}\right) \boldsymbol{G}, \\
s_{t}^{\varepsilon}+\operatorname{div}_{\boldsymbol{x}}\left[\boldsymbol{v}_{T}^{\varepsilon} f\left(s^{\varepsilon}\right)\right]+\frac{\partial}{\partial y}\left[\lambda_{\mathrm{o}}(s) f(s)\left(\rho_{\mathrm{w}}-\rho_{\mathrm{o}}\right)\right]=0, \\
\nabla_{\boldsymbol{x}} \Lambda_{i} \cdot \boldsymbol{n}=0, i=\mathrm{o}, \mathrm{w}, \\
\nabla_{\boldsymbol{x}} p \cdot \boldsymbol{n}=0, \\
\nabla_{\boldsymbol{x}} s^{\varepsilon} \cdot \boldsymbol{n}=0, \\
s(0, \boldsymbol{x})=s_{0}(\boldsymbol{x}), \quad t>0, \quad \boldsymbol{x} \in \partial \Omega \Omega \\
\boldsymbol{x} \in \Omega,
\end{gathered}
$$

respectively.

It is straightforward to define schemes for the three models, (2.14), (2.15) and (2.16), by combining the schemes (2.5), (2.6), (2.7) and (2.8) with appropriate boundary conditions. As before, we have that for $\varepsilon>0$, the schemes produce approximate solutions for (2.15) and (2.16) which converge as $\Delta x \downarrow 0$.

We have tested the schemes on a $150 \times 300$ grid $\left(\Delta x=\Delta y\right.$, for $\varepsilon=10^{-2}$ and $\varepsilon=10^{-5}$. Figure 4 shows the approximations for $t=0.3, t=0.6$ and $t=0.9$ using $\varepsilon=10^{-2}$ We see that the Darcy model and the Helmholtz model produce fairly similar results, but that results produced by the Brinkman model are very different. It remains to investigate whether this holds for the much smaller $\varepsilon=10^{-5}$.

Figure 5 shows the same simulation results as Figure 4 , but with $\varepsilon=10^{-5}$. We 

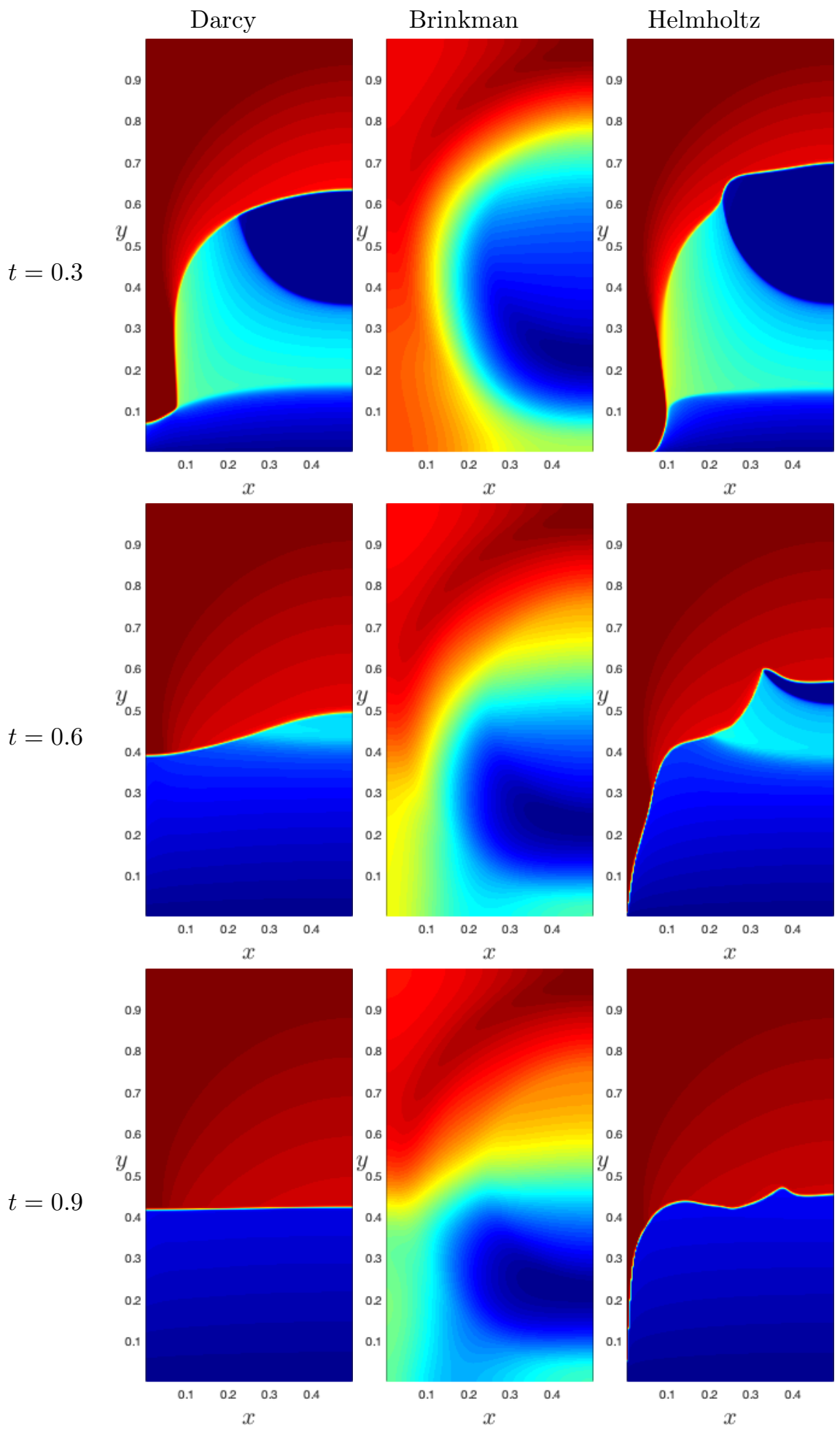

Figure 4. Approximate solutions with gravity driven flow. $\varepsilon=10^{-2}$.

see that for $\varepsilon=10^{-5}$, the Darcy model and the Helmholtz model produce virtually identical results, but again, the Brinkman model behaves differently. There are 

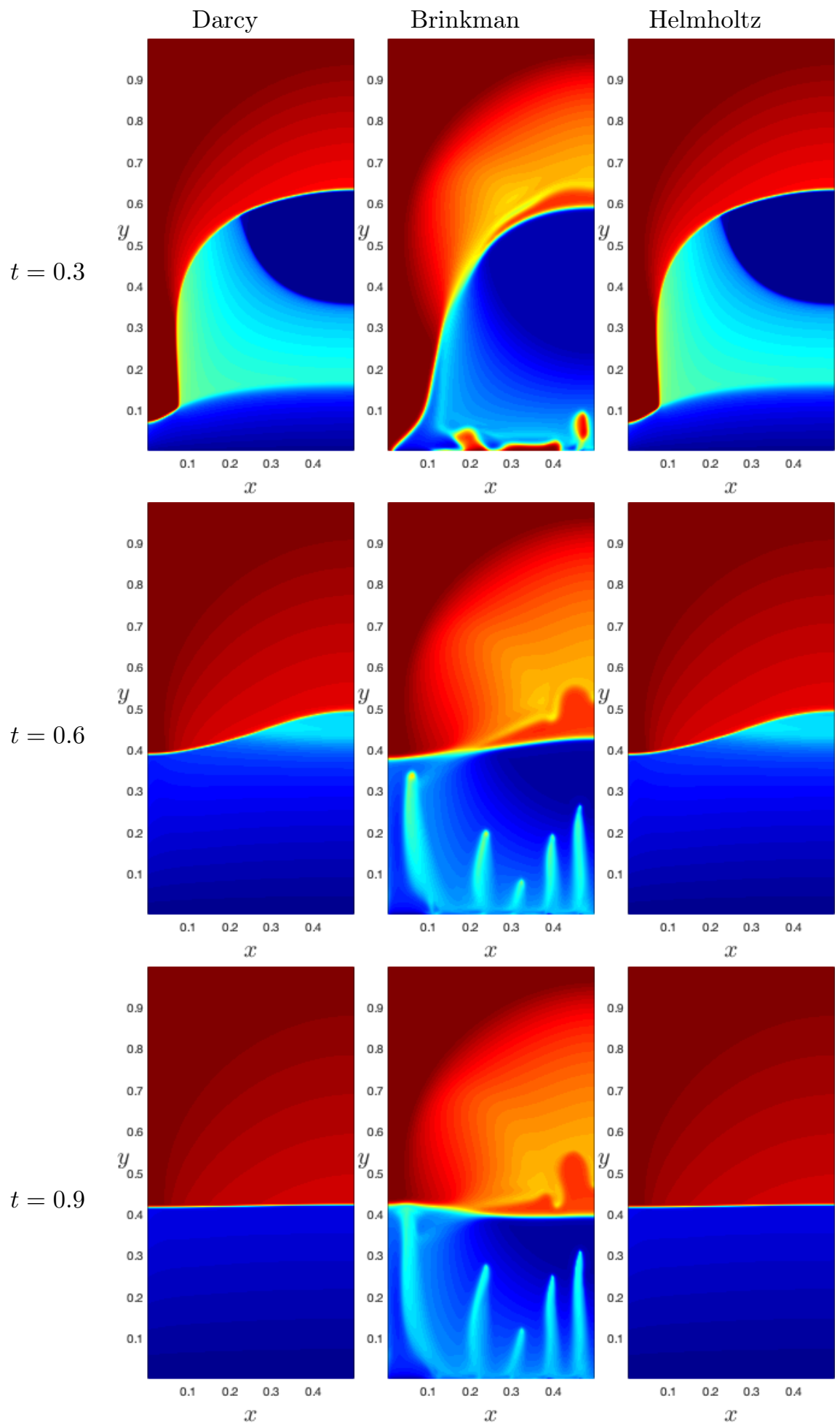

FigURE 5. Approximate solutions with gravity driven flow. $\varepsilon=10^{-5}$.

several visible fine scale results, reminiscent of the characteristic "viscous fingering" often seen in simulations using the Darcy model. Such viscous fingering is usually 
attributed to heterogeneous rock permeability affecting the (probable) intrinsic instability in the model. From Figure 5 we can conclude that the Brinkman model with homogeneous rock properties can produce similar results.

Remark. To remain physically meaningful, the saturation $s$ should remain inside the interval $[0,1]$. One can show that both the standard Darcy model (1.8) and the Helmholtz model (1.13) have $[0,1]$ as an invariant region for the saturation. For the Brinkman model (1.11) this is not the case, as has been demonstrated for one-dimensional examples in [9]. In the numerical examples in this paper the calculations for the Brinkman model always gave nonnegative saturations, but some values were larger than one. It is not clear how to augment the Brinkman model so that $[0,1]$ becomes an invariant region for the saturation.

\section{REFERENCES}

[1] Jørg E. Aarnes, Tore Gimse, and Knut-Andreas Lie, An introduction to the numerics of flow in porous media using Matlab, Geometric modelling, numerical simulation, and optimization: applied mathematics at SINTEF, Springer, Berlin, 2007, pp. 265-306. MR 2348925

[2] Adimurthi, Siddhartha Mishra, and G. D. Veerappa Gowda, Conservation law with the flux function discontinuous in the space variable. II. Convex-concave type fluxes and generalized entropy solutions, J. Comput. Appl. Math. 203 (2007), no. 2, 310-344. MR 2323047

[3] Boris Andreianov, Kenneth Hvistendahl Karlsen, and Nils Henrik Risebro, A theory of $L^{1}$ dissipative solvers for scalar conservation laws with discontinuous flux, Arch. Ration. Mech. Anal. 201 (2011), no. 1, 27-86.

[4] Alaa Armiti-Juber and Christian Rohde, On Darcy-and Brinkman-Type Models for TwoPhase Flow in Asymptotically Flat Domains, arXiv e-prints (2017), arXiv:1712.07470.

[5] H. C. Brinkman, A calculation of the viscous force exerted by a flowing fluid on a dense swarm of particles, Flow, Turbulence and Combustion 1 (1949), no. 1, 27-34.

[6] Gui-Qiang Chen and Kenneth H. Karlsen, Quasilinear anisotropic degenerate parabolic equations with time-space dependent diffusion coefficients, Commun. Pure Appl. Anal. 4 (2005), no. 2, 241-266.

[7] G. M. Coclite, K. H. Karlsen, S. Mishra, and N. H. Risebro, A hyperbolic-elliptic model of two-phase flow in porous media-existence of entropy solutions, Int. J. Numer. Anal. Model. 9 (2012), no. 3, 562-583.

[8] G. M. Coclite, S. Mishra, and N. H. Risebro, Convergence of an Engquist-Osher scheme for a multi-dimensional triangular system of conservation laws, Math. Comp. 79 (2010), no. 269, $71-94$.

[9] G. M. Coclite, S. Mishra, N. H. Risebro, and F. Weber, Analysis and numerical approximation of Brinkman regularization of two-phase flows in porous media, Comput. Geosci. 18 (2014), no. $5,637-659$.

[10] Giuseppe Maria Coclite and Nils Henrik Risebro, Conservation laws with time dependent discontinuous coefficients, SIAM J. Math. Anal. 36 (2005), no. 4, 1293-1309.

[11] Robert Eymard, Raphaèle Herbin, and Anthony Michel, Mathematical study of a petroleumengineering scheme, M2AN Math. Model. Numer. Anal. 37 (2003), no. 6, 937-972.

[12] Tore Gimse and Nils Henrik Risebro, Solution of the Cauchy problem for a conservation law with a discontinuous flux function, SIAM J. Math. Anal. 23 (1992), no. 3, 635-648.

[13] Helge Holden and Nils Henrik Risebro, Front tracking for hyperbolic conservation laws, second ed., Applied Mathematical Sciences, vol. 152, Springer, Heidelberg, 2015.

[14] K. H. Karlsen, N. H. Risebro, and J. D. Towers, $L^{1}$ stability for entropy solutions of nonlinear degenerate parabolic convection-diffusion equations with discontinuous coefficients, Skr. K. Nor. Vidensk. Selsk. (2003), no. 3, 1-49.

[15] Kenneth Hvistendahl Karlsen and Nils Henrik Risebro, Convergence of finite difference schemes for viscous and inviscid conservation laws with rough coefficients, M2AN Math. Model. Numer. Anal. 35 (2001), no. 2, 239-269.

[16] Dietmar Kroener and Stephan Luckhaus, Flow of oil and water in a porous medium, J. Differential Equations 55 (1984), no. 2, 276-288.

[17] S. N. Kruzhkov and S. M. Sukorjanskiı̌, Boundary value problems for systems of equations of two-phase filtration type; formulation of problems, questions of solvability, justification of approximate methods, Mat. Sb. (N.S.) 104(146) (1977), no. 1, 69-88, 175-176.

[18] Philippe G. LeFloch, Hyperbolic systems of conservation laws, Lectures in Mathematics ETH Zürich, Birkhäuser Verlag, Basel, 2002. 
[19] S. Lukkhaus and P. I. Plotnikov, Entropy solutions of Buckley-Leverett equations, Sibirsk. Mat. Zh. 41 (2000), no. 2, 400-420, iv.

[20] S. P. Neuman, Theoretical derivation of Darcy's law, Acta Mechanica 25 (1977), no. 3-4, 153-170.

[21] Felix Otto, Stability investigation of planar solutions of Buckley-Leverett equation, Tech. report, Sonderforchungbereich 256, 1995, Preprint no. 345.

[22] Donald W. Peaceman, Fundamentals of numerical reservoir simulation, Elsevier Science Inc., New York, NY, USA, 1991.

(Nils Henrik Risebro)

DePARTMENT OF MATHEMATics

UNIVERSITY OF OSLO

P.O. Box 1053, BLindern

N-0316 Oslo, Norway

E-mail address: nilsr@math.uio.no 